\title{
Technical note: Glucose concentration in dairy cows measured using 6 handheld meters designed for human use
}

\author{
R. B. Lopes, ${ }^{1}$ A. Valldecabres, ${ }^{1}$ and N. Silva-del-Río ${ }^{1,2 *}$ \\ ${ }^{1}$ Veterinary Medicine Teaching and Research Center, 18830 Road 112, Tulare, CA 93274 \\ ${ }^{2}$ Department of Population Health and Reproduction, School of Veterinary Medicine, University of California, Davis 95616
}

\section{ABSTRACT}

The objective was to evaluate the precision and accuracy of 6 handheld glucose meters, designed for human use [Accu-Chek Aviva Plus (AC), Roche Diabetes Care, Mannheim, Germany; Aga Matrix (AM), AgaMatrix Inc., Salem, NH; Contour Next (CT), Bayer HealthCare LLC, Leverkusen, Germany; FreeStyle Precision Neo (FS), Abbott Diabetes Care Ltd., Alameda, CA; Nova Max Plus (NM), Nova Biomedical Corporation, Waltham, MA; and Precision Xtra (PX), Abbott Diabetes Care Ltd., Witney, UK] to measure blood glucose concentration in dairy cows. Blood samples from Jersey and Jersey $\times$ Holstein crossbreed cows $(\mathrm{n}=97$ for all; except $\mathrm{CT}, \mathrm{n}=71$ ) were collected and analyzed in triplicate using the 6 handheld glucose meters evaluated. Plasma glucose was also measured with the laboratory reference method (hexokinase glucose-6-phosphate dehydrogenase). Based on the intra-assay coefficient of variation $(\mathrm{CV})$, precision varied across handheld glucose meters: AC (2.2\%), CT (4.0\%), PX (4.7\%), FS (5.6\%), AM (6.2\%), and NM (6.7\%). Lin's concordance correlation coefficients between handheld glucose meters and the reference method were 0.75 for FS, 0.74 for PX, 0.62 for $\mathrm{AC}, 0.55$ for $\mathrm{CT}, 0.53$ for $\mathrm{NM}$, and 0.48 for AM. Based on Passing-Bablok regression, the AM and PX meters showed bias in the measurements of blood glucose. Bland-Altman plots indicated a negative bias $(\mathrm{FS}=-0.25 \mathrm{mmol} / \mathrm{L} ; \mathrm{CT}=-0.60 \mathrm{mmol} / \mathrm{L})$ or a positive bias $(\mathrm{AM}=0.29 \mathrm{mmol} / \mathrm{L} ; \mathrm{PX}=0.33 \mathrm{mmol} / \mathrm{L}$; $\mathrm{NM}=0.52 \mathrm{mmol} / \mathrm{L} ; \mathrm{AC}=0.65 \mathrm{mmol} / \mathrm{L})$ between handheld glucose meters and the reference method. All handheld glucose meters evaluated had wide limits of agreement (LoA) ranging from -0.18 to $1.47 \mathrm{mmol} / \mathrm{L}$ (AC, narrowest LoA) to -1.25 to $1.82 \mathrm{mmol} / \mathrm{L}$ (AM, widest LoA). Bias was the major contributor to the total observed error $\left(\mathrm{TE}_{\mathrm{obs}}\right)$, accounting for $81.5 \%$ of the $\mathrm{TE}_{\text {obs }}$ in $\mathrm{AC}, 72.0 \%$ in $\mathrm{CT}, 64.9 \%$ in $\mathrm{AM}, 61.1 \%$ in $\mathrm{NM}$, $57.8 \%$ in PX, and $56.2 \%$ in FS. Overall, although some

Received September 13, 2018.

Accepted June 4, 2019.

*Corresponding author: nsilvadelrio@ucdavis.edu handheld meters (AC, CT, and PX) showed satisfactory precision, none were accurate measuring glucose. Future studies should evaluate whether incorporating algorithms designed for cattle can improve accuracy and precision of handheld glucose meters.

Key words: handheld meter, glucose, dairy cow

\section{Technical Note}

Glucose concentration has been proposed as a plausible biomarker for health and reproduction of postpartum dairy cows (Senosy et al., 2012; Garverick et al., 2013; Bicalho et al., 2017). Garverick et al. (2013) found that at 3 wk postpartum cows with blood glucose concentration $>3.33 \mathrm{mmol} / \mathrm{L}$ had a greater chance of pregnancy at first insemination than cows with lower blood glucose concentration $<2.22 \mathrm{mmol} / \mathrm{L}$ (40 vs. $21 \%$ ). Furthermore, blood glucose concentration has been associated with impaired health, mainly in transition cows. Gordon et al. (2017) observed a differential treatment response to hyperketonemia based on cows' glycemic status. During the early postpartum period, one-third of hyperketonemic cows are also hypoglycemic (blood glucose $\leq 2.22 \mathrm{mmol} / \mathrm{L}$; Dubuc and Buczinski, 2018); thus, monitoring programs for ketosis might benefit from testing glucose concentrations of hyperketonemic cows. Senosy et al. (2012) showed that at 4 wk postpartum, cows with subclinical endometritis had lower blood glucose concentration than healthy cows (2.73 vs. $2.98 \mathrm{mmol} / \mathrm{L})$. However, Bicalho et al. (2017) observed that higher blood glucose concentrations at $3 \mathrm{~d}$ postpartum can be a predictor for metritis $(>3.10$ $\mathrm{mmol} / \mathrm{L})$ and clinical endometritis $(>2.49 \mathrm{mmol} / \mathrm{L})$. More studies are necessary to establish a reference interval for glucose concentrations in healthy dairy cows at various physiological and productive stages.

Some research studies have used handheld meters designed for glucose monitoring in diabetic human patients to measure cow-side blood glucose concentrations (Galvão et al., 2010; Gordon et al., 2017; Ruoff et al., 2017). These meters are appealing because they are easy to procure and more affordable and convenient than laboratory analysis. Many such meters are available on 
the market, but the number of studies validating their use in dairy cows is limited (Wittrock et al., 2013; Mair et al., 2016; Macmillan et al., 2017). The objective of the present study was to evaluate the precision and accuracy of 6 handheld meters, designed for monitoring of blood glucose in humans, to measure blood glucose concentration in dairy cows.

All procedures were approved by the University of California Davis Institutional Animal Care and Use Committee (\#18846). A method-agreement study was conducted to evaluate 6 handheld glucose meters: Accu-Chek Aviva Plus (AC; Roche Diabetes Care, Mannheim, Germany), AgaMatrix Presto (AM; AgaMatrix Inc., Salem, NH), Contour Next (CT; Bayer HealthCare LLC, Leverkusen, Germany), FreeStyle Precision Neo (FS; Abbott Diabetes Care Ltd., Alameda, CA), Nova Max Plus (NM; Nova Biomedical Corporation, Waltham, MA), and Precision Xtra (PX; Abbott Diabetes Care Ltd., Witney, UK). Sample size calculation was based on Mair et al. (2016); from the Bland-Altman plot, the mean difference between a handheld glucose meter and the reference method was $0.26 \mathrm{mmol} / \mathrm{L}$, with a standard deviation of the difference of $0.34 \mathrm{mmol} / \mathrm{L}$. A total of 95 cows were deemed necessary (type I error $=0.05$, type II error $=0.2$ ) to detect a difference of $1.11 \mathrm{mmol} / \mathrm{L}$ in glucose concentration between methods (Wittrock et al., 2013). Ninety-seven cows were enrolled from September to October 2016 (3 $\mathrm{d}$ of enrollment); 26, 28, and 43 cows were enrolled per study day. The study herd was located in California and milked 8,932 Jersey and Jersey $\times$ Holstein crossbreed cows with an average milk yield of $24.3 \mathrm{~kg} / \mathrm{d}$. To achieve a wide range of glucose concentrations, cows at different productive stages were selected from 3 groups: close-up ( $\mathrm{n}=29$ for all, except for CT $=20)$, fresh (n $=54$ for all, except $\mathrm{CT}=37)$, and high-producing (n $=14$ for all). Dry cows were moved to close-up pens $21 \mathrm{~d}$ before expected calving date. After parturition, cows were moved to a postpartum pen, transferred to a fresh-cow pen where they stayed for $30 \mathrm{~d}$, and subsequently moved to a high-producing lactation pen.

Cows enrolled in the study were sampled twice from the coccygeal vessels. Two blood specimens were collected, the first into a $3-\mathrm{mL}$ syringe and the second into a Vacutainer tube containing sodium fluoride and potassium oxalate (Becton Dickinson and Co., Franklin Lakes, NJ). This sampling method was adopted to expedite glucose testing with handheld meters; the syringe allowed quick placement of a drop of blood onto the test strip. To ensure a similar ratio of arterial and venous blood, blood specimens collected from the 2 puncture sites were evaluated for color difference. If differences in color were detected, samples were collected again. This sampling protocol was validated using 20 lactating dairy cows. Following the same protocol as described above, blood samples were collected from the coccygeal vessels into Vacutainer tubes containing sodium fluoride and potassium oxalate at 2 puncture sites and kept on ice for $40 \mathrm{~min}$ until centrifugation $(1,500 \times$ g, $20 \mathrm{~min}, 15^{\circ} \mathrm{C}$ ). Plasma harvested from each blood specimen was aliquoted into 2 microcentrifuge tubes, frozen at $-20^{\circ} \mathrm{C}$ for $1 \mathrm{~d}$, and shipped to the laboratory for glucose analyses [Marshfield Lab, Marshfield, WI; hexokinase glucose-6-phosphate dehydrogenase (G-6-PDH) method]. The coefficient of variation (CV) for the duplicated plasma aliquots from the same blood specimen (intra-assay CV) was $0.7 \%$ and that for blood specimens obtained from the 2 puncture sites was $1.1 \%$.

Immediately after specimens were collected, a drop of blood from the $3-\mathrm{mL}$ syringe was placed on the electrochemical test strip of each handheld glucose meter. This procedure was repeated 3 times within 5 min after sample collection. Handheld glucose meters and test strips were handled according to manufacturer specifications, as described in Table 1. Before use, all glucose meters and test strip lots were tested using a control solution. In this technology, blood glucose reacts with enzymes inside the electrochemical test strips, either glucose oxidase (AM and NM) or glucose dehydrogenase (CT, FS, PX, and AC), and produces an electrical current (amperometry) or charge (coulometry) that is converted into glucose concentration (Aggidis and Newman, 2015). Because all handheld meters displayed glucose in milligrams per deciliter $(\mathrm{mg} / \mathrm{dL})$, a conversion factor was used to present the data in millimoles per liter (glucose in $\mathrm{mg} / \mathrm{dL} \times 0.0555=$ glucose in $\mathrm{mmol} / \mathrm{L}$ ). The second blood samples, collected in the sodium fluoride and potassium oxalate tubes, were kept on ice for an average of $3 \mathrm{~h} 30 \mathrm{~min}$ until centrifugation at $1,430 \times g$ for $15 \mathrm{~min}$ at $12^{\circ} \mathrm{C}$. Based on the previously reported stability of glucose concentration in refrigerated whole-blood samples coated with sodium fluoride and potassium oxalate (Morris et al., 2002) or lithium heparin (Valldecabres et al., 2018), we considered the handling protocol of blood specimens adequate for glucose analysis. Plasma was harvested and stored at $-20^{\circ} \mathrm{C}$ for $23 \mathrm{~d}$ until analysis. Frozen plasma samples were then shipped to Marshfield Lab and analyzed for glucose concentration using the hexokinase G-6-PDH method (glucose reagents SR6621, OSR6121, and OSR6221; Beckman Coulter Inc., Brea, CA) using an automatic analyzer (AU5800, Beckman Coulter Inc.). These results were used as the reference method or gold standard. The intra- and interassay laboratory CV for glucose were 0.4 and $0.7 \%$, respectively.

To evaluate the precision of the handheld glucose meters, triplicate measurements were used to calculate the intra-assay CV $[($ mean/SD $) \times 100]$ using Excel 
Table 1. Specifications of the 6 handheld glucose meters and test strips

\begin{tabular}{lcccl}
\hline $\begin{array}{l}\text { Handheld } \\
\text { meter }^{1}\end{array}$ & $\begin{array}{c}\text { Assay range } \\
(\mathrm{mmol} / \mathrm{L})\end{array}$ & $\begin{array}{c}\text { Sample } \\
\text { volume }(\mu \mathrm{L})\end{array}$ & $\begin{array}{c}\text { Test strip storage } \\
\text { temperature }\left({ }^{\circ} \mathrm{C}\right)\end{array}$ & $\begin{array}{l}\text { Test strip lot } \\
\text { number }\end{array}$ \\
\hline $\mathrm{AC}$ & $0.50-33.30$ & 0.6 & $2-30$ & 496244 \\
$\mathrm{AM}$ & $1.10-33.30$ & 0.5 & $8-30$ & MB07WD03G \\
$\mathrm{CT}$ & $0.50-33.30$ & 0.6 & $9-30$ & DW6DJ302B \\
$\mathrm{FS}$ & $1.10-27.75$ & 0.6 & $4-30$ & 4500166934 \\
$\mathrm{NM}$ & $1.10-33.30$ & 0.3 & $15-30$ & 1020216041 \\
$\mathrm{PX}$ & $1.10-27.75$ & 0.6 & $4-30$ & 4500167328 \\
\hline
\end{tabular}

${ }^{1}$ Accu-Chek Aviva Plus (AC; Roche Diabetes Care, Mannheim, Germany), AgaMatrix Presto (AM; AgaMatrix Inc., Salem, NH), Contour Next (CT; Bayer HealthCare LLC, Leverkusen, Germany), FreeStyle Precision Neo (FS; Abbott Diabetes Care Ltd., Alameda, CA), Nova Max Plus (NM; Nova Biomedical Corporation, Waltham, MA), and Precision Xtra (PX; Abbott Diabetes Care Ltd., Witney, UK).

spreadsheets (2010; Microsoft Corp., Redmond, WA). Statistical analyses were performed using MedCalc version 17.8.6 (MedCalc Software bvba, Ostend, Belgium) and SigmaPlot version 14.0 (Systat Software Inc., San Jose, CA). Data were tested for normality with the Shapiro-Wilk test and for linearity with the Cusum test. The agreement between glucose concentration obtained with the reference method and that obtained using the handheld glucose meters was evaluated using Lin's concordance correlation coefficients $(\mathbf{C C C} ; \boldsymbol{\rho c})$, where $-1=$ perfect disagreement and $1=$ perfect agreement (Carrasco et al., 2013), and BlandAltman plots (Bland and Altman, 1986). Replicated glucose measurements from the handheld meters were averaged and plotted against glucose measurements obtained with the reference method (Myles and Cui, 2007). Normal distribution of the bias (bias = reference method - mean of handheld meter) was evaluated with the Shapiro-Wilk test. Passing-Bablok regression was performed to evaluate the presence of constant and proportional bias (Passing and Bablok, 1983). The performance of handheld glucose meters was evaluated using a modified calculation of the total observed error $\left(\mathbf{T E}_{\mathrm{obs}}\right)$ proposed by Harr et al. (2013) as follows: $\mathrm{TE}_{\text {obs }}=2 \times$ intra-assay CV (\%) + bias (\%), where bias was obtained as [(reference method - mean of handheld meter $) /$ reference method] $\times 100$.
A total of 97 blood samples were used to evaluate 5 of the 6 handheld glucose meters. Because of the lack of supplies, CT was evaluated using only 71 blood samples. In our study, some blood specimens coagulated before all 3 glucose measurements were performed. The proportion of glucose readings lost because of coagulation was $19.9 \%(\mathrm{AC}), 19.2 \%(\mathrm{AM}), 24.4 \%(\mathrm{CT}), 18.6 \%$ (FS), $18.2 \%(\mathrm{NM})$, and $12.7 \%(\mathrm{PX})$. In addition, some glucose measurements produced a reading error $[\mathrm{AC}$ (6.0\%), AM (4.3\%), CT (3.1\%), FS (2.5\%), NM (2.9\%), and PX $(0.8 \%)]$. The 3 most frequent types of error observed were incorrect test procedure (placing a larger or smaller drop in the test strip than required), outof-range hematocrit and glucose levels, and electronic error (Table 2). Plasma glucose measured with the reference method from all 97 cows ranged from 1.89 to $6.05 \mathrm{mmol} / \mathrm{L}(3.41 \pm 0.71 \mathrm{mmol} / \mathrm{L} ;$ mean $\pm \mathrm{SD})$. Descriptive statistics of the glucose measurements obtained with the 6 handheld meters are shown in Table 3.

The intra-assay CV of glucose samples measured with the handheld meters are presented in Figure 1. For clinical use, handheld glucose meters should have a CV $<5 \%$ (Skeie et al., 2001). In the present study, the CV was below this threshold for AC (2.2\%), CT (4.0\%), and PX (4.7\%) but higher for FS (5.6\%), AM (6.2\%), and NM (6.7\%). To the best of our knowledge, 6 studies

Table 2. Description and frequency of error types when using 6 handheld glucose meters in dairy cows

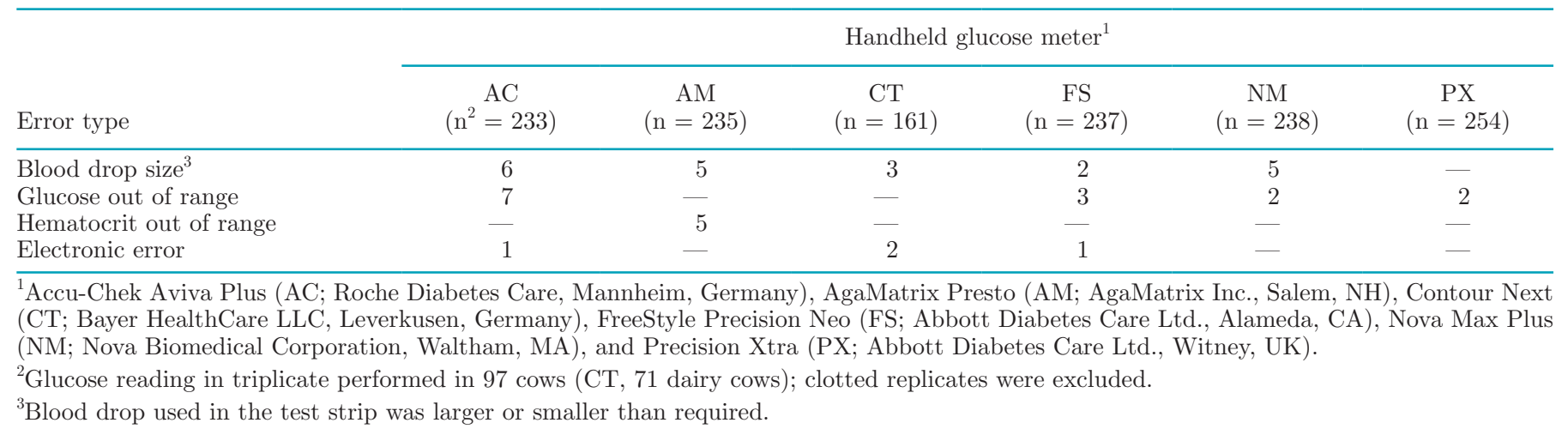


have evaluated the suitability of handheld meters for glucose determination in dairy cows, but only 2 of those (Megahed et al., 2015; Mair et al., 2016) reported the intra-assay CV. In these studies, 3 (Mair et al., 2016) and 2 (Megahed et al., 2015) blood samples with different glucose concentrations were replicated 10 times. Two of the meters included in our study were also evaluated by Mair et al. (2016; FS, intra-assay CV = $5.5 \%$ ) and Megahed et al. (2015; PX, intra-assay CV = $5.0 \%)$. Those studies reported CV inferior to what we observed; however, differences in methodology, such as the number of samples and replicates, may be partially responsible for these observations.

According to Lin's CCC, FS $(\rho c=0.75)$ and PX $(\rho c$ $=0.74)$ gave results in strong agreement with reference method, whereas AC $(\rho c=0.62)$, CT $(\rho c=0.55)$, $\mathrm{NM}(\rho \mathrm{c}=0.53)$, and AM $(\rho \mathrm{c}=0.48)$ gave results in moderate agreement. The PX data did not follow a normal distribution; however, according to Lin (1989), CCC may be robust even with non-normal data. In our study, the CCC for FS was superior $(\rho \mathrm{c}=0.75)$ to the 0.56 reported by Macmillan et al. (2017). In previous studies, the degree of association between handheld glucose meters and the reference method was evaluated with Pearson correlation coefficients, resulting in very strong ( $\mathrm{r}=0.95$, Wittrock et al., 2013), strong ( $\mathrm{r}=$ 0.80 , Mair et al., 2016), or moderate $(r=0.63$, Voyvoda and Erdogan, 2010; r = 0.41, Mair et al., 2016) associations. However, Pearson correlation evaluates the association between 2 methods, whereas CCC quantifies the agreement among them (Lin, 1989).

The presence of constant and proportional bias was studied with Passing-Bablok regressions (Figure 2). Four handheld glucose meters (CT, FS, NM, and AC) showed no indication of constant or proportional bias based on regression analysis; however, AM showed a proportional bias (slope did not include 1) and PX a constant and proportional bias (intercept did not in-

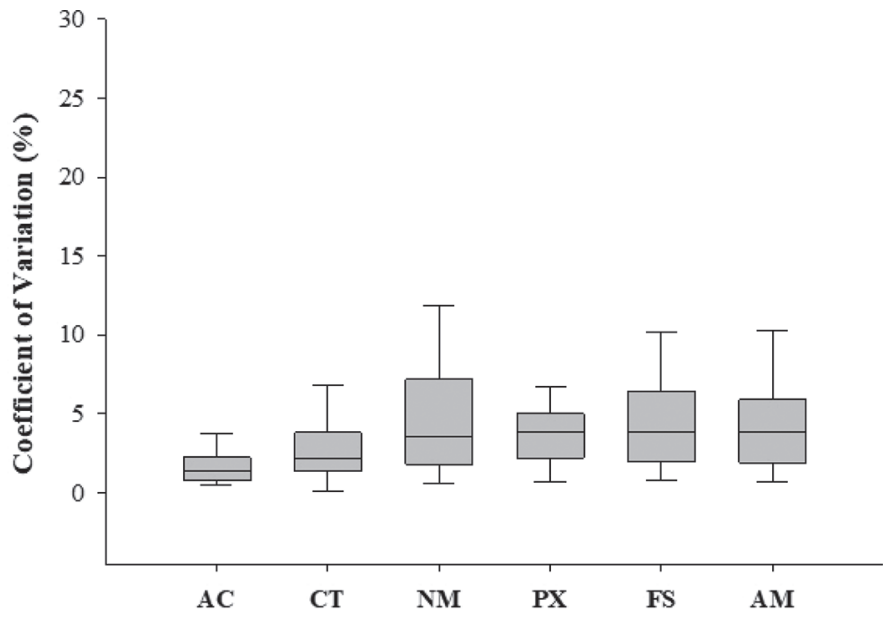

Figure 1. Boxplot distribution of the intra-assay coefficient of variation $(\mathrm{CV})$ of the 6 handheld glucose meters for glucose concentrations: Accu-Chek Aviva Plus (AC; Roche Diabetes Care, Mannheim, Germany), Contour Next (CT; Bayer HealthCare LLC, Leverkusen, Germany), FreeStyle Precision Neo (FS; Abbott Diabetes Care Ltd., Alameda, CA), AgaMatrix Presto (AM; AgaMatrix Inc., Salem, NH), Precision Xtra (PX; Abbott Diabetes Care Ltd., Witney, UK), and Nova Max Plus (NM; Nova Biomedical Corporation, Waltham, MA). Each boxplot shows the 50th percentile (median, line within the box), 25th and 75th percentiles (box), and 10th and 90th percentiles (whiskers). Data are presented sorted by ascending CV mean.

clude 0 and slope did not include 1 ). It should be noted that Passing-Bablok regression does not account for random variation. Therefore, agreement between methods was further evaluated using Bland-Altman plots (Figure 3$)$. The bias $(\mathrm{mmol} / \mathrm{L})$ observed was negative for FS $(-0.25)$ and $\mathrm{CT}(-0.60)$ and positive for AM (0.29), PX (0.33), NM (0.52), and AC (0.65). The difference between methods fell within acceptable limits of agreement (LoA; $1.96 \mathrm{SD})$ at least $95 \%$ of the time for PX (96.7\%), AM (95.8\%), and CT (95.7\%); however, it was less than $95 \%$ for FS (94.8\%), NM (94.8\%), and AC (94.8\%). Previous studies assessed the agreement

Table 3. Descriptive statistics of glucose concentrations (mmol/L) obtained after using 6 handheld meters (triplicate measurements) and the reference method (Laboratory) in 97 dairy cows (CT, 71 dairy cows) ${ }^{1}$

\begin{tabular}{lcccccc}
\hline $\begin{array}{l}\text { Handheld }_{\text {meter }^{2}} \\
\text { CT }\end{array}$ & $\begin{array}{c}\text { Cows } \\
\text { (n) }\end{array}$ & Mean & Median & SD & Minimum & Maximum \\
\hline FS & 71 & 2.79 & 2.76 & 0.59 & 1.56 & 5.22 \\
Laboratory & 97 & 3.16 & 3.07 & 0.72 & 1.68 & 5.85 \\
AM & 97 & 3.41 & 3.33 & 0.72 & 1.89 & 6.05 \\
PX & 97 & 3.70 & 3.68 & 0.86 & 1.39 & 6.44 \\
NM & 97 & 3.75 & 3.62 & 0.95 & 1.96 & 8.05 \\
AC & 97 & 3.94 & 3.85 & 0.71 & 1.48 & 6.03 \\
\hline
\end{tabular}

${ }^{1}$ Data are presented sorted in ascending order based on the mean.

${ }^{2}$ Accu-Chek Aviva Plus (AC; Roche Diabetes Care, Mannheim, Germany), AgaMatrix Presto (AM; AgaMatrix Inc., Salem, NH), Contour Next (CT; Bayer HealthCare LLC, Leverkusen, Germany), FreeStyle Precision Neo (FS; Abbott Diabetes Care Ltd., Alameda, CA), Nova Max Plus (NM; Nova Biomedical Corporation, Waltham, MA), and Precision Xtra (PX; Abbott Diabetes Care Ltd., Witney, UK). 

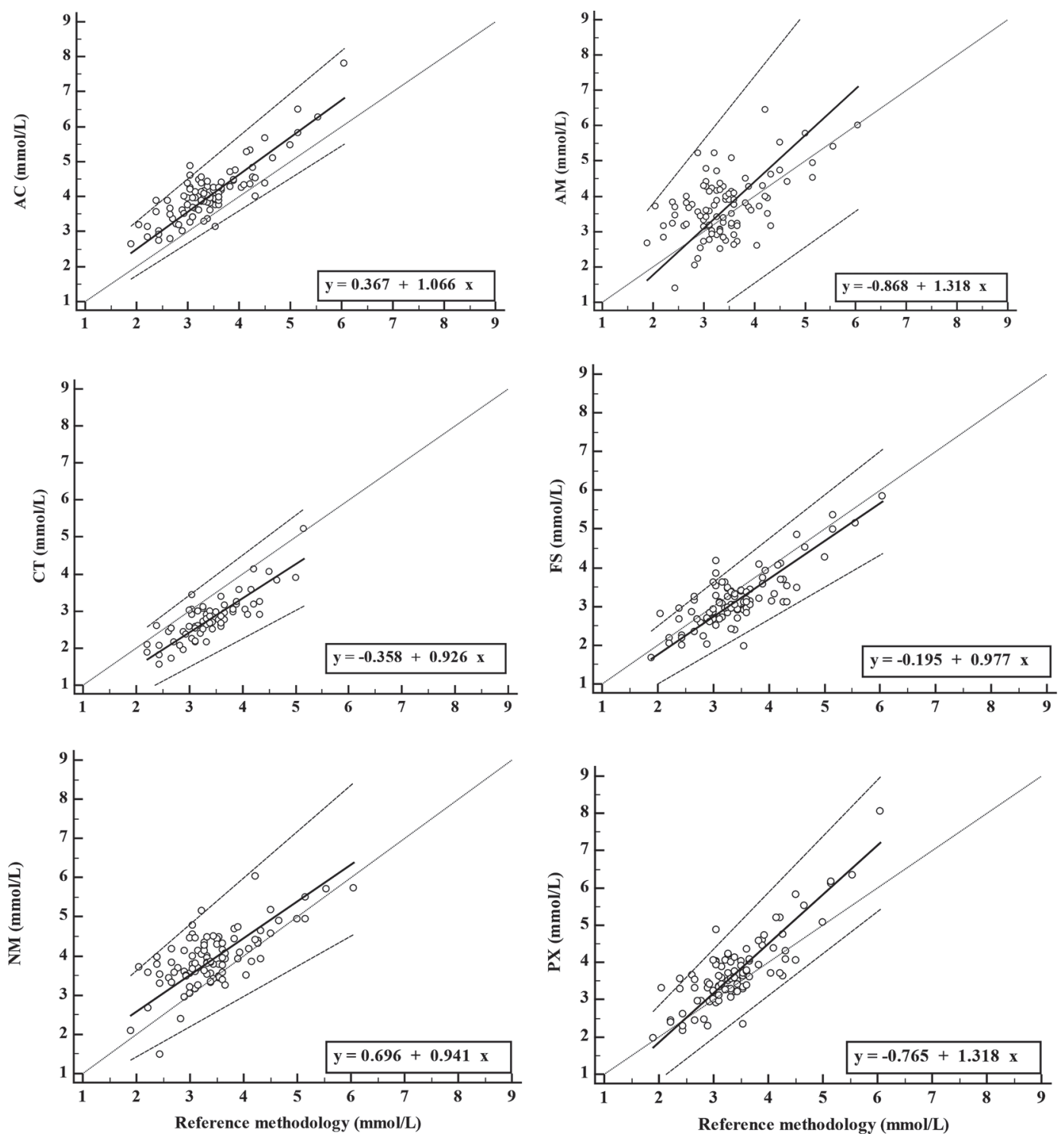

Figure 2. Passing-Bablok regression for glucose concentrations from Accu-Chek Aviva Plus (AC; Roche Diabetes Care, Mannheim, Germany), Contour Next (CT; Bayer HealthCare LLC, Leverkusen, Germany), FreeStyle Precision Neo (FS; Abbott Diabetes Care Ltd., Alameda, CA), AgaMatrix Presto (AM; AgaMatrix Inc., Salem, NH), Precision Xtra (PX; Abbott Diabetes Care Ltd., Witney, UK), and Nova Max Plus (NM; Nova Biomedical Corporation, Waltham, MA) against the reference method. The solid line indicates the Passing-Bablok regression line, upper and lower lines are the regression confidence limits $(95 \%)$, and the dotted line is the identity line $(\mathrm{x}=\mathrm{y})$. Panels are presented sorted alphabetically.

of FS (Macmillan et al., 2017) and PX (Wittrock et al., 2013); similar to our results, 95 and $98 \%$ of samples in those studies were within the LoA. In the present study, all 6 handheld meters showed LoA that were wide enough to bring into question their suitability as a diagnostic tool. The LoA ranged from -0.18 to 1.47 $\mathrm{mmol} / \mathrm{L}$ (AC, narrowest LoA) and from -1.25 to 1.82 $\mathrm{mmol} / \mathrm{L}$ (AM, widest LoA). In our study, FS had the second narrowest LoA $(-1.15$ to $0.65 \mathrm{mmol} / \mathrm{L})$, with values comparable to previous results for this handheld meter (LoA: -1.11 to $0.55 \mathrm{mmol} / \mathrm{L}$; Mair et al., 2016).
The modified calculation of $\mathrm{TE}_{\mathrm{obs}}$ of handheld glucose meters is shown in Figure 4. Bias was the major contributor to $\mathrm{TE}_{\mathrm{obs}}$, accounting for $81.5 \%$ of the $\mathrm{TE}_{\mathrm{obs}}$ in $\mathrm{AC}, 72.0 \%$ in $\mathrm{CT}, 64.9 \%$ in $\mathrm{AM}, 61.1 \%$ in NM, $57.8 \%$ in PX, and $56.2 \%$ in FS. Based on guidelines from the American Society for Veterinary Clinical Pathology (ASVCP), instrument performance is considered acceptable if, after evaluating quality control material on 5 different days, $\mathrm{TE}_{\mathrm{obs}} \leq 20 \%$ (Harr et al., 2013). In our study, triplicate glucose measurements resulted in a $\mathrm{TE}_{\mathrm{obs}} \leq 20 \%$ on $54.6 \%$ of samples for PX, $50.5 \%$ for 
FS, $48.5 \%$ for AC, $37.1 \%$ for AM, $36.6 \%$ for $\mathrm{CT}$, and $35.1 \%$ for NM. Most of the samples exceeded the recommended allowable total error, suggesting poor analytical performance of most handheld glucose meters.

All handheld glucose meters evaluated in the present study were designed for human use and intended as monitoring tools for diabetic patients. In human blood specimens, hematocrit interferes with glucose concentration readings (Vanavanan et al., 2010). However, the hematocrit of cows is lower than that of humans; therefore, evaluating glucose concentration with handheld meters designed for humans may result in an overestimation of glucose concentration (Vanavanan et al., 2010). None of the handheld meters used in the present study corrected glucose readings for hematocrit. It has been proposed that handheld glucose meters designed for human use might perform better if they were calibrated or adjusted for cattle measurements (Megahed
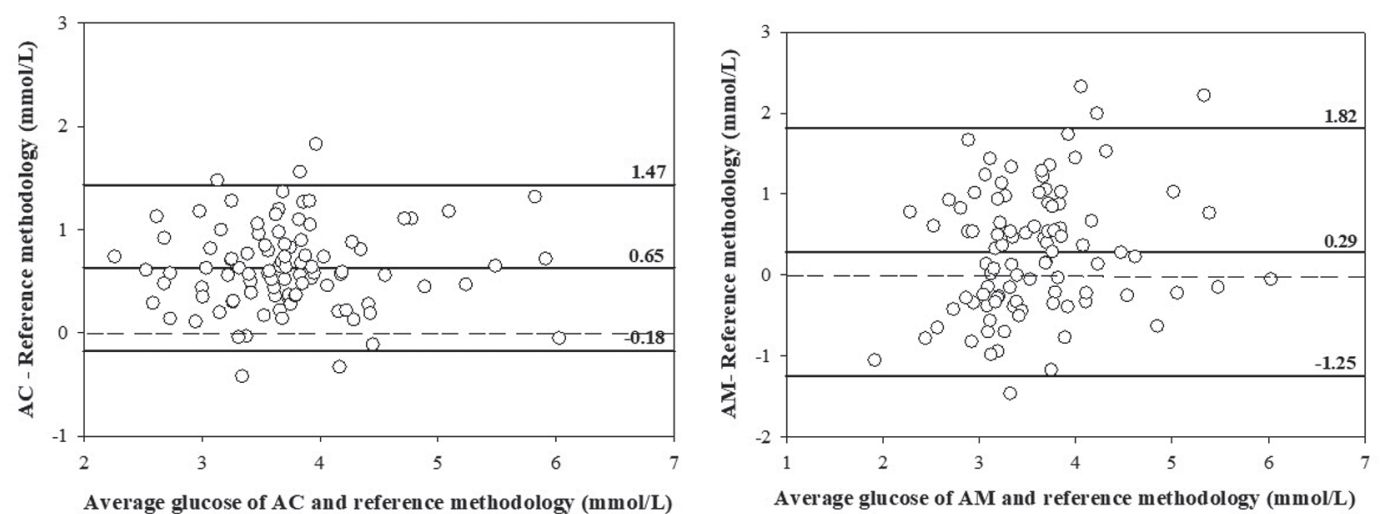

Average glucose of $\mathrm{AC}$ and reference methodology (mmol/L)
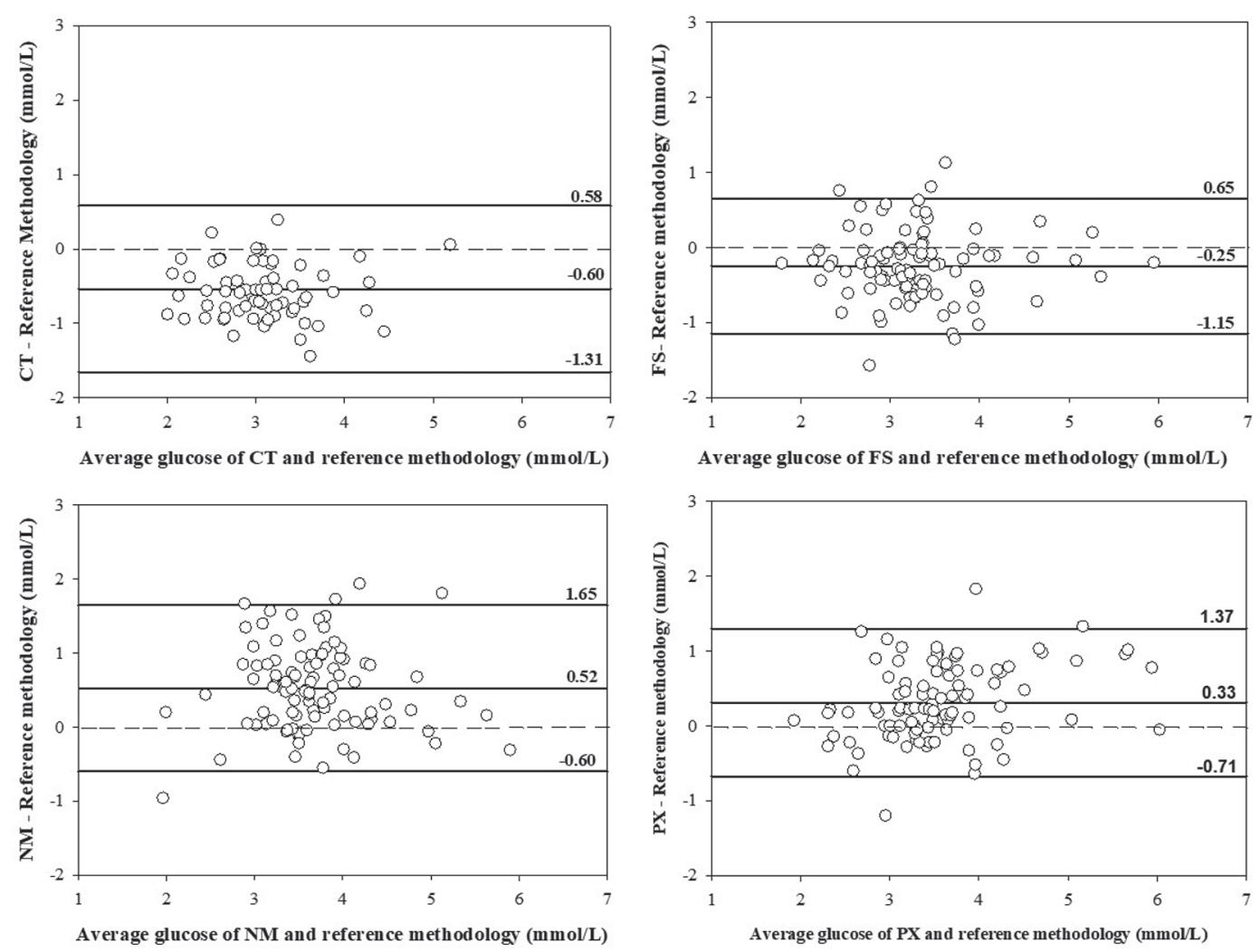

Figure 3. Bland-Altman plot of differences in glucose concentrations analyzed with the reference method in plasma and measured in whole blood with Accu-Chek Aviva Plus (AC; Roche Diabetes Care, Mannheim, Germany), Contour Next (CT; Bayer HealthCare LLC, Leverkusen, Germany), FreeStyle Precision Neo (FS; Abbott Diabetes Care Ltd., Alameda, CA), AgaMatrix Presto (AM; AgaMatrix Inc., Salem, NH), Precision Xtra (PX; Abbott Diabetes Care Ltd., Witney, UK), and Nova Max Plus (NM; Nova Biomedical Corporation, Waltham, MA). The solid line in the middle shows the mean (bias); the upper and lower lines represent the limit of agreement (bias $\pm 1.96 \mathrm{SD}$ ), and the dashed line indicates bias $=0$. Panels are presented sorted alphabetically. Data are presented in alphabetical order. 
et al., 2015). Some manufacturers offer handheld glucose meters targeting animal species including cattle. Although $\beta$-hydroxybutyrate handheld meters designed for cattle have been evaluated (Kanz et al., 2015; Bach et al., 2016), to the best of our knowledge, no study has evaluated handheld glucose meters marketed for use in cattle or incorporating algorithms designed for cattle. A potential limitation of the present study is that blood specimens were collected from coccygeal vessels even though the handheld glucose meters evaluated are designed for capillary whole blood. Nevertheless, a previous study showed insignificant bias between coccygeal blood from venous and capillary vessels (Mair et al., 2016).

The present study used different specimens - whole blood for the handheld glucose meters and plasma for the reference method. These differences between specimens might have introduced a constant bias but it was not detected based on Passing-Bablok regression results. In humans, glucose concentration is 7.3 to $10.0 \%$ lower in whole blood than in plasma (Kuwa et al., 2001; Higioka et al., 2016). Furthermore, storage of frozen plasma for $23 \mathrm{~d}$ before analysis could have affected glucose concentrations, but there are limiting and conflicting results supporting this premise (Clark et al., 1990; Morris et al., 2002; Wu et al., 2017). In addition, 2 of the handheld glucose meters evaluated (AM and NM) used the glucose oxidase method that is sensitive to $\mathrm{pH}$, which was not evaluated in the present study. However, the implications of blood $\mathrm{pH}$ for glucose readings when using handheld glucose meters are unknown (Gerber and Freeman, 2016).

In our study, important differences in performance were noted across the 6 handheld glucose meters evaluated. Precision was acceptable in some handheld glucose meters (AC, CT, and PX). Passing-Bablok regression showed that AM had a constant bias and PX had a systematic and constant bias. Based on Bland-Altman plots, none of the handheld glucose meters were accurate. According to American Society for Veterinary Clinical Pathology guidelines, only $54.6 \%$ (PX) to $35.1 \%$ (NM) of the glucose readings had a $\mathrm{TE}_{\mathrm{obs}} \leq 20 \%$. Thus, based on accuracy, none of the handheld glucose meters evaluated can be considered adequate to measure glucose in dairy cows. Future studies should evaluate whether incorporating algorithms designed

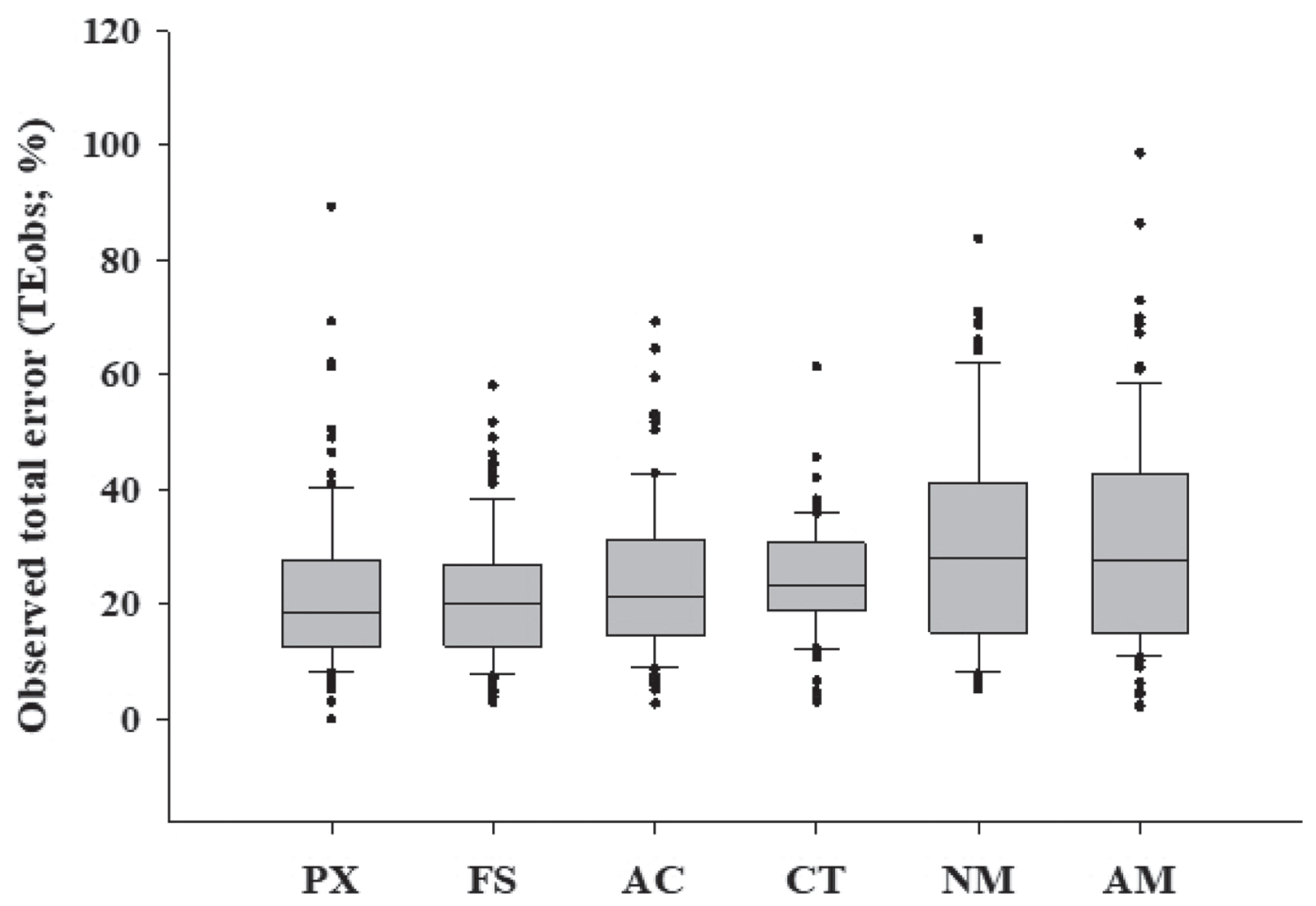

Figure 4. Boxplot distribution of the total observed error $\left[\mathrm{TE}_{\mathrm{obs}}=2 \times\right.$ intra-assay $\mathrm{CV}(\%)+$ Bias $\left.(\%)\right]$, where bias was obtained as $[($ reference method - mean of handheld meter)/reference method] $\times 100$ of 6 handheld glucose meters: Accu-Chek Aviva Plus (AC; Roche Diabetes Care, Mannheim, Germany), Contour Next (CT; Bayer HealthCare LLC, Leverkusen, Germany), FreeStyle Precision Neo (FS; Abbott Diabetes Care Ltd., Alameda, CA), AgaMatrix Presto (AM; AgaMatrix Inc., Salem, NH), Precision Xtra (PX; Abbott Diabetes Care Ltd., Witney, UK), and Nova Max Plus (NM; Nova Biomedical Corporation, Waltham, MA). Each boxplot shows the 50th percentile (median, line within the box), 25th and 75th percentiles (box), 10th and 90th percentiles (whiskers), and outliers (dots). Data are presented sorted by the TE $\mathrm{obs}_{\text {mean. }}$ 
for cattle into handheld glucose meters will result in greater accuracy than that currently available in handheld meters used for glucose monitoring in humans.

\section{ACKNOWLEDGMENTS}

No financial support was received for the research or publication of this work. The authors appreciate the collaboration of Pacific Rim Dairy (Corcoran, CA) in allowing us to use their facilities and cows.

\section{REFERENCES}

Aggidis, A. G., and J. D. Newman. 2015. Investigating pipeline and state of the art blood glucose biosensors to formulate next steps. Biosens. Bioelectron. 74:243-262. https://doi.org/10.1016/ j.bios.2015.05.071.

Bach, K. D., W. Heuwieser, and J. A. A. McArt. 2016. Comparison of 4 electronic handheld meters for diagnosing hyperketonemia in dairy cows. J. Dairy Sci. 99:9136-9142. https://doi.org/10.3168/ jds.2016-11077.

Bicalho, M. L. S., E. C. Marques, R. O. Gilbert, and R. C. Bicalho 2017. The association of plasma glucose, BHBA, and NEFA with postpartum uterine diseases, fertility, and milk production of Holstein dairy cows. Theriogenology 88:270-282. https://doi.org/10 $.1016 / \mathrm{j}$.theriogenology.2016.09.036.

Bland, J. M., and D. G. Altman. 1986. Statistical methods for assessing agreement between two methods of clinical measurement. Lancet 327:307-310. https://doi.org/10.1016/S0140-6736(86)90837-8.

Carrasco, J. L., B. R. Phillips, J. Puig-Martinez, T. S. King, and V. M. Chinchilli. 2013. Estimation of the concordance correlation coefficient for repeated measures using SAS and R. Comput. Methods Programs Biomed. 109:293-304. https://doi.org/10.1016/j.cmpb 2012.09.002.

Clark, M. L., S. M. Humphreys, and K. N. Frayn. 1990. Stability of plasma glucose during storage. Ann. Clin. Biochem. 27:373-377. https://doi.org/10.1177/000456329002700415.

Dubuc, J., and S. Buczinski. 2018. Cow-and herd-level prevalence of hypoglycemia in hyperketonemic postpartum dairy cows. J. Dairy Sci. 101:3374-3379. https://doi.org/10.3168/jds.2017-13773.

Galvão, K. N., M. J. B. F. Flaminio, S. B. Brittin, R. Sper, M. Fraga, L. Caixeta, A. Ricci, C. L. Guard, W. R. Butler, and R. O. Gilbert. 2010. Association between uterine disease and indicators of neutrophil and systemic energy status in lactating Holstein cows. J. Dairy Sci. 93:2926-2937. https://doi.org/10.3168/jds.2009-2551.

Garverick, H. A., M. N. Harris, R. Vogel-Bluel, J. D. Sampson, J. Bader, W. R. Lamberson, J. N. Spain, M. C. Lucy, and R. S. Youngquist. 2013. Concentrations of nonesterified fatty acids and glucose in blood of periparturient dairy cows are indicative of pregnancy success at first insemination. J. Dairy Sci. 96:181-188. https://doi.org/10.3168/jds.2012-5619.

Gerber, K. L., and K. P. Freeman. 2016. ASVCP guidelines: Quality assurance for portable blood glucose meter (glucometer) use in veterinary medicine. Vet. Clin. Pathol. 45:10-27. https://doi.org/ 10.1111/vcp. 12310 .

Gordon, J. L., T. F. Duffield, T. H. Herdt, D. F. Kelton, L. Neuder, and S. J. LeBlanc. 2017. Effects of a combination butaphosphan and cyanocobalamin product and insulin on ketosis resolution and milk production. J. Dairy Sci. 100:2954-2966. https://doi.org/10 $.3168 /$ jds.2016-11925.

Harr, K. E., B. Flatland, M. Nabity, and K. P. Freeman. 2013. ASVCP guidelines: Allowable total error guidelines for biochemistry. Vet. Clin. Pathol. 42:424-436. https://doi.org/10.1111/vcp.12101.

Higioka, A. S., R. C. M. Rudolf-Oliveira, F. Martinello, and M. L. Bazzo. 2016. Analytical performance of glucometers in a tertiary care hospital. Accredit. Qual. Assur. 21:143-149. https://doi.org/ 10.1007/s00769-015-1188-2.
Kanz, P., M. Drillich, D. Klein-Jöbstl, B. Mair, S. Borchardt, L. Meyer, and M. Iwersen. 2015. Suitability of capillary blood obtained by a minimally invasive lancet technique to detect subclinical ketosis in dairy cows by using 3 different electronic hand-held devices. J. Dairy Sci. 98:6108-6118. https://doi.org/10.3168/jds.2014-8957.

Kuwa, K., T. Nakayama, T. Hoshino, and M. Tominaga. 2001. Relationships of glucose concentrations in capillary whole blood, venous whole blood and venous plasma. Clin. Chim. Acta 307:187192. https://doi.org/10.1016/S0009-8981(01)00426-0.

Lin, L. I.-K. 1989. A concordance correlation coefficient to evaluate reproducibility. Biometrics 45:255-268. https://doi.org/10.2307/ 2532051

Macmillan, K., I. L. Helguera, A. Behrouzi, M. Gobikrushanth, B. Hoff, and M. G. Colazo. 2017. Accuracy of a cow-side test for the diagnosis of hyperketonemia and hypoglycemia in lactating dairy cows. Res. Vet. Sci. 115:327-331. https://doi.org/10.1016/j.rvsc 2017.06.019.

Mair, B., M. Drillich, D. Klein-Jöbstl, P. Kanz, S. Borchardt, L. Meyer, I. Schwendenwein, and M. Iwersen. 2016. Glucose concentration in capillary blood of dairy cows obtained by a minimally invasive lancet technique and determined with three different hand-held meters. BMC Vet. Res. 12:34-44. https://doi.org/10.1186/s12917 $-016-0662-3$

Megahed, A. A., M. W. H. Hiew, J. R. Townsend, J. B. Messick, and P. D. Constable. 2015. Evaluation of an electrochemical point of care meter for measuring glucose concentration in blood from periparturient dairy cattle. J. Vet. Intern. Med. 29:1718-1727. https: //doi.org/10.1111/jvim.13608.

Morris, J. D., J. M. Fernandez, A. M. Chapa, L. R. Gentry, K. E. Thorn, and T. M. Weick. 2002. Effects of sample handling, processing, storage, and hemolysis on measurements of key energy metabolites in ovine blood. Small Rumin. Res. 43:157-166. https: //doi.org/10.1016/S0921-4488(01)00266-8.

Myles, P. S., and J. Cui. 2007. I. Using the Bland-Altman method to measure agreement with repeated measures. Br. J. Anaesth. 99:309-311. https://doi.org/10.1093/bja/aem214.

Passing, H., and W. Bablok. 1983. A new biometrical procedure for testing the equality of measurements from two different analytical methods. Application of linear regression procedures for method comparison studies in clinical chemistry, Part I. J. Clin. Chem. Clin. Biochem. 21:709-720. https://doi.org/10.1515/cclm.1983.21 .11.709.

Ruoff, J., S. Borchardt, and W. Heuwieser. 2017. Associations between blood glucose concentration, onset of hyperketonemia, and milk production in early lactation dairy cows. J. Dairy Sci. 100:54625467. https://doi.org/10.3168/jds.2016-12237.

Senosy, W. S., Y. Izaike, and T. Osawa. 2012. Influences of metabolic traits on subclinical endometritis at different intervals postpartum in high milking cows. Reprod. Domest. Anim. 47:666-674. https:/ /doi.org/10.1111/j.1439-0531.2011.01941.x.

Skeie, S., G. Thue, and S. Sandberg. 2001. Patient-derived quality specifications for instruments used in self-monitoring of blood glucose. Clin. Chem. 47:67-73.

Valldecabres, A., R. Lopes, and N. Silva-del-Río. 2018. Ionized calcium and glucose changes in refrigerated heparinized blood samples from dairy cows. J. Dairy Sci. 101(E-Suppl. 2):84. (Abstr.)

Vanavanan, S., P. Santanirand, U. Chaichanajarernkul, A. Chittamma, J. A. DuBois, T. Shirey, and M. Heinz. 2010. Performance of a new interference-resistant glucose meter. Clin. Biochem. 43:186192. https://doi.org/10.1016/j.clinbiochem.2009.09.010.

Voyvoda, H., and H. Erdogan. 2010. Use of a hand-held meter for detecting subclinical ketosis in dairy cows. Res. Vet. Sci. 89:344-351. https://doi.org/10.1016/j.rvsc.2010.04.007.

Wittrock, J. A. M., T. F. Duffield, and S. J. LeBlanc. 2013. Validation of a point-of-care glucometer for use in dairy cows. J. Dairy Sci. 96:4514-4518. https://doi.org/10.3168/jds.2012-6533.

Wu, D. W., Y. M. Li, and F. Wang. 2017. How long can we store blood samples: A systematic review and meta-analysis. EBioMedicine 24:277-285. https://doi.org/10.1016/j.ebiom.2017.09.024. 